INSTITUT NATIONAL DE RECHERCHE EN INFORMATIQUE ET EN AUTOMATIQUE

\title{
The game Grundy number of graphs
}

\author{
Frédéric Havet — Xuding Zhu
}

$\mathbf{N}^{\circ} 7646$

June 2011

Thème COM

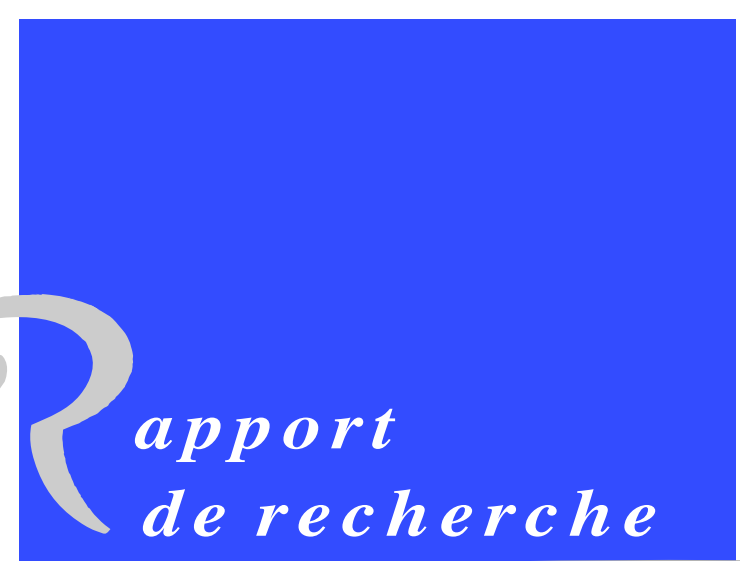





\title{
The game Grundy number of graphs
}

\author{
Frédéric Havet ${ }^{*}$, Xuding Zhu $\dagger^{\dagger}$ \\ Thème COM - Systèmes communicants \\ Équipe-Projet Mascotte \\ Rapport de recherche $n^{\circ} 7646$ - June 2011 - 16 pages
}

\begin{abstract}
Given a graph $G=(V, E)$, two players, Alice and Bob, alternate their turns in choosing uncoloured vertices to be coloured. Whenever an uncoloured vertex is chosen, it is coloured by the least positive integer not used by any of its coloured neighbours. Alice's goal is to minimize the total number of colours used in the game, and Bob's goal is to maximize it. The game Grundy number of $G$ is the number of colours used in the game when both players use optimal strategies. It is proved in this paper that the maximum game Grundy number of forests is 3 , and the game Grundy number of any partial 2-tree is at most 7 .
\end{abstract}

Key-words: colouring game, game Grundy number, trees, partial 2-trees

* Projet Mascotte, I3S (CNRS, UNS) and INRIA, Sophia Antipolis. Partly supported by the French Agence Nationale de la Recherche under Grant GRATEL ANR-09-blan-0373-01. Email: Frederic.Havet@inria.fr

$\dagger$ Department of Mathematics, Zhejiang Normal University, China. Grant number: ZJNSF No. Z6110786. Email: xudingzhu@gmail.com 


\section{Le nombre Grundy par jeu des graphes}

Résumé : Etant donné un graphe $G=(V, E)$, deux joueurs, Alice et Bob, jouent chacun leur tour en choisissant un sommet non-coloré. A chaque fois, qu'un sommet est choisi, il est coloré avec le plus petit entier naturel qui n'est assigné à aucun de ses voisins. Le but d'Alice est de minimiser le nombre de couleurs utilisées, et le but de Bob est de maximiser ce nombre. Le nombre Grundy par jeu de $G$ est le nombre de couleurs utilisées si les deux joueurs adoptent un stratégie optimale. Dans ce rapport, nous montrons que le nombre Grundy par jeu maximal d'une forêt est 3 et que le nombre Grundy par jeu d'un 2 -arbre partiel est au plus 7 .

Mots-clés : jeu de coloration, nombre Grundy par jeu, arbre, 2-arbre partiel 


\section{Introduction}

Suppose $G=(V, E)$ is a graph. The game chromatic numbers of $G$ are defined through a two-person game: the colouring game. Let $X$ be a set of colours. Alice and Bob take turns in playing the game. Each play by either player consists of colouring an uncoloured vertex of $G$ with a colour from $X$. Adjacent vertices must be coloured by distinct colours. The game ends if no more vertices can be coloured: either because all the vertices are coloured or because the uncoloured vertices have no legal colour, i.e., for each uncoloured vertex $x$, each colour from $X$ is used by a neighbour of $x$. Alice wins the game if all the vertices of $G$ are successfully coloured. Otherwise, Bob wins the game. The Alice-first colouring game is the colouring game when Alice has the first move and Bob-first colouring game is the colouring game when Bob has the first move. The Alice-first game chromatic number $\chi_{\mathrm{g}}^{A}(G)$ (resp. Bob-first game chromatic number $\chi_{\mathrm{g}}^{B}(G)$ ) of $G$ is the least number of colours in a colour set $X$ for which Alice has a winning strategy in the Alice-first (resp. Bob-first) colouring game on $G$.

For classes $\mathcal{H}$ of graphs, the Alice-first game chromatic number and Bob-first game chromatic number of $\mathcal{H}$ are defined as respectively $\chi_{\mathrm{g}}^{A}(\mathcal{H})=\max \left\{\chi_{\mathrm{g}}^{A}(G)\right.$ : $G \in \mathcal{H}\}$ and $\chi_{\mathrm{g}}^{B}(\mathcal{H})=\max \left\{\chi_{\mathrm{g}}^{B}(G): G \in \mathcal{H}\right\}$. Although for a single graph $G$, there can be a big difference between $\chi_{\mathrm{g}}^{A}(G)$ and $\chi_{\mathrm{g}}^{B}(G)$, for natural classes of graphs, we usually have $\chi_{\mathrm{g}}^{A}(\mathcal{H})=\chi_{\mathrm{g}}^{B}(\mathcal{H})$. For a graph $G$, let $2 G$ denote the union of two disjoint copies of $G$, and $G^{+}$denote the graph obtained from $G$ by adding an isolated vertex. The following proposition was proved in [20].

Proposition 1. Suppose $\mathcal{H}$ is a class of graphs such that if $G \in \mathcal{H}$, then $2 G \in \mathcal{H}$ and $G^{+} \in \mathcal{H}$. Then $\chi_{\mathrm{g}}^{A}(\mathcal{H})=\chi_{\mathrm{g}}^{B}(\mathcal{H})$.

Therefore when studying such graph classes it is sufficient to consider Alicefirst game chromatic number. For simplicity reasons and as very often in the literature, we shall abbreviate Alice-first game chromatic in game chromatic number and write $\chi_{\mathrm{g}}$ instead of $\chi_{\mathrm{g}}^{A}$.

The colouring game on planar maps was invented by Brams, and was published by Gardner [6] in his column "Mathematical Games" in Scientific American in 1981. It remained unnoticed by the graph-theoretic community until ten years later, when it was reinvented by Bodlaender [2]. Bodlaender defined the game chromatic number of graphs, and conjectured that the game chromatic number of planar graphs is bounded by a constant. Since then the problem has attracted considerable attention and the game chromatic numbers of various classes of graphs have been studied (see [1] for a recent survey).

We denote by $\mathcal{F}$ the family of forests, by $\mathcal{I}_{k}$ the family of interval graphs with clique number $k$, by $\mathcal{P}$ the family of planar graphs, by $\mathcal{Q}$ the family of outerplanar graphs, by $\mathcal{P} \mathcal{T}_{k}$ the family of partial $k$-trees. It is proved by Faigle, Kern, Kierstead and Trotter [5] that $\chi_{\mathrm{g}}(\mathcal{F})=4$, proved by Faigle, Kern, Kierstead and Trotter [5] that $\chi_{\mathrm{g}}\left(\mathcal{I}_{k}\right) \leq 3 k-2$, proved by Guan and Zhu [7] and Kierstead and Trotter [10] that $6 \leq \chi_{\mathrm{g}}(\mathcal{Q}) \leq 7$, and proved by Zhu [18, that $\chi_{\mathrm{g}}\left(\mathcal{P} \mathcal{T}_{k}\right) \leq 3 k+2$ for $k \geq 2$, proved by Zhu [19] and Kierstead and Trotter [10] that $8 \leq \chi_{\mathrm{g}}(\mathcal{P}) \leq 17$.

$\mathrm{RR} \mathrm{n}^{\circ} 7646$ 
To obtain upper bounds for the game chromatic number of graphs, one usually study another game: the marking game. Suppose $G=(V, E)$ is a graph. In the marking game on $G$, two players, Alice and Bob, take turns (with Alice having the first move) marking an unmarked vertex of $G$. The game ends when all vertices are marked. For a vertex $x$ of $G$, let $b(x)$ be the number of neighbours of $x$ that are marked before $x$ is marked. The score of the game is

$$
s=1+\max _{x \in V(G)} b(x) .
$$

Alice's goal is to minimize the score, while Bob's goal is to maximize it. The game colouring number $\operatorname{col}_{\mathrm{g}}(G)$ of $G$ is the least $s$ such that Alice has a strategy that results in a score at most $s$ in the marking game on $G$. For a family $\mathcal{H}$ of graphs, $\operatorname{col}_{\mathrm{g}}(\mathcal{H})=\max \left\{\operatorname{col}_{\mathrm{g}}(G): G \in \mathcal{H}\right\}$.

The game colouring number of a graph was first formally introduced in [17] as a tool in the study of the game chromatic number of graphs. However, it is of independent interest. The concept itself and some of its variations have been studied extensively in the literature [1, 3, 4, 7, 8, 9, 11, 12, 13, 14, 15, 16, 18, 19,.

The exact value of the game colouring numbers of $\mathcal{F}, \mathcal{I}_{k}, \mathcal{Q}$ and $\mathcal{P} \mathcal{T}_{k}$ are known. It is proved by Faigle, Kern, Kierstead and Trotter [5] that $\operatorname{col}_{g}(\mathcal{F})=4$, proved by Faigle, Kern, Kierstead and Trotter [5] and Kierstead and Yang [14] that $\operatorname{col}_{\mathrm{g}}\left(\mathcal{I}_{k}\right)=3 k-2$, proved by Guan and Zhu 7] and Kierstead and Yang [14 that $\operatorname{col}_{\mathrm{g}}(\mathcal{Q})=7$, and proved by Zhu [18] and $\mathrm{Wu}$ and Zhu [16] that $\operatorname{col}_{\mathrm{g}}\left(\mathcal{P} \mathcal{T}_{k}\right)=3 k+2$ for $k \geq 2$. It is also known [19, 16] that $11 \leq \operatorname{col}_{\mathrm{g}}(\mathcal{P}) \leq 17$.

It is easy to see that for any graph $G, \chi_{\mathrm{g}}(G) \leq \operatorname{col}_{\mathrm{g}}(G)$ and for many natural classes of graphs, the best known upper bounds for their game chromatic numbers are obtained by finding upper bounds for their game colouring numbers.

In this paper, we introduce a game, the greedy colouring game. In some sense, this new game is a mixture of the colouring game and the marking game. Suppose $G=(V, E)$ is a graph. Two players, Alice and Bob, alternate their turns in choosing an uncoloured vertex and colour it greedily, that is with the minimum positive integer not already assigned to one of its coloured neighbours. The aim of Alice is to minimize the number of used colours and the aim of Bob is to maximize it. Again, there are two greedy colouring games depending wether Alice or Bob has the first move. Assume both players use their optimal strategies, the number of colours used at the end of the game is called the Alicefirst game Grundy number of the graph if Alice plays first, and the Bob-first game Grundy number if Bob plays first. It is denoted by $\Gamma_{\mathrm{g}}^{A}(G)$ and $\Gamma_{\mathrm{g}}^{B}(G)$ respectively. At the end, the obtained colouring is a greedy colouring so the two game Grundy numbers are greater than or equal to the chromatic number and less or equal to the Grundy number.

For classes $\mathcal{H}$ of graphs, the Alice-first game Grundy number and Bob-first game Grundy number of $\mathcal{H}$ are defined as respectively $\Gamma_{\mathrm{g}}^{A}(\mathcal{H})=\max \left\{\Gamma_{\mathrm{g}}^{A}(G)\right.$ : $G \in \mathcal{H}\}$ and $\Gamma_{\mathrm{g}}^{B}(\mathcal{H})=\max \left\{\Gamma_{\mathrm{g}}^{B}(G): G \in \mathcal{H}\right\}$. 
Similarly to game chromatic numbers, the two numbers $\Gamma_{\mathrm{g}}^{A}(G)$ and $\Gamma_{\mathrm{g}}^{B}(G)$ may differ a lot, for example for the graph $K_{n, n}^{*}$ obtained from the complete bipartite graph $K_{n, n}$ by removing a perfect matching.

Proposition 2. If $n \geq 2$, then $\Gamma_{g}^{A}\left(K_{n, n}^{*}\right)=n$ and $\Gamma_{\mathrm{g}}^{B}\left(K_{n, n}^{*}\right)=2$.

Proof. Suppose that Alice starts, then Bob will always play on the vertex joined to the one Alice played by an edge of the removed matching. It is then easy to see that $n$ colours are used, one per edge of the removed matching. Hence $\Gamma_{\mathrm{g}}^{A}\left(K_{n, n}^{*}\right) \geq n$. It is well known that $\Gamma\left(K_{n, n}^{*}\right)=n$, so $\Gamma_{\mathrm{g}}^{A}\left(K_{n, n}^{*}\right)=n$.

Suppose now that Bob starts. Let us denote by $\left(V_{1}, V_{2}\right)$ the bipartition of $K_{n, n}^{*}$. Bob first colour one vertex $v$ with 1 . Without loss of generality, $v \in V_{1}$. Then Alice can play on another vertex $w$ of $V_{1}$. This vertex will be coloured 1 . Now every vertex of $V_{2}$ is adjacent to either $v$ or $w$, so no vertex of $V_{2}$ will be coloured 1. Thus each time a player will choose a vertex of $V_{1}$, he will colour it with 1 , since it has no neighbour coloured 1 . Hence at the end all the vertices of $V_{1}$ will be coloured 1 . Therefore, each time a player chooses a vertex of $V_{2}$, he will colour it with 2 , since it is only adjacent to vertices coloured 1 . So all the vertices of $V_{2}$ will be coloured 2 . So $\Gamma_{\mathrm{g}}^{B}\left(K_{n, n}^{*}\right) \geq 2$. Since $\chi\left(K_{n, n}^{*}\right)=2$, we have $\Gamma_{\mathrm{g}}^{B}\left(K_{n, n}^{*}\right)=2$.

In the above proposition Alice-first game Grundy number is larger than Bob-first game Grundy number, but it can be the converse. Consider the graph $H=\left(K_{n, n}^{*}\right)^{+}$. Then the first player could play on the isolated vertex and then apply the strategy of Proposition 2 as second player. Hence $\Gamma_{\mathrm{g}}^{A}(H)=2$ and $\Gamma_{\mathrm{g}}^{B}(H)=n$.

Suppose $\mathcal{H}$ is a class of graphs. Let $\Gamma_{\mathrm{g}}^{A}(\mathcal{H})=\max \left\{\Gamma_{\mathrm{g}}^{A}(G): G \in \mathcal{H}\right\}$, and $\Gamma_{\mathrm{g}}^{B}(\mathcal{H})=\max \left\{\Gamma_{\mathrm{g}}^{B}(G): G \in \mathcal{H}\right\}$. Similarly to Proposition 1, for natural classes of graphs, we usually have $\Gamma_{\mathrm{g}}^{A}(\mathcal{H})=\Gamma_{\mathrm{g}}^{B}(\mathcal{H})$.

Proposition 3. Suppose $\mathcal{H}$ is a class of graphs such that if $G \in \mathcal{H}$, then $2 G \in \mathcal{H}$ and $G^{+} \in \mathcal{H}$. Then $\Gamma_{\mathrm{g}}^{A}(\mathcal{H})=\Gamma_{\mathrm{g}}^{B}(\mathcal{H})$.

Proof. Assume $\Gamma_{\mathrm{g}}^{B}(\mathcal{H})=k$. Then there is a graph $G \in \mathcal{H}$ such that $\Gamma_{\mathrm{g}}^{B}(G)>k-$ 1, i.e., Bob has a strategy, called BWIN-Strategy, to ensure that the maximum colour used in the greedy colouring game is at least $k$.

Assume first that $G$ has an odd number of vertices. We shall show that $\Gamma_{\mathrm{g}}^{A}(2 G)>k-1$. Let the two copies of $G$ in $2 G$ be $G_{1}$ and $G_{2}$. Assume Alice colours a vertex of $G_{1}$ in her first move. Then Bob colours a vertex of $G_{2}$ in his first move, according to BWIN-Strategy. From then on, whenever Alice colours a vertex of $G_{2}$, Bob also colours a vertex of $G_{2}$, using BWIN-Strategy. If Alice colours a vertex of $G_{1}$, then Bob colours an arbitrary vertex of $G_{1}$. Because $G_{1}$ has an odd number of vertices, whenever Alice colours a vertex of $G_{1}$, Bob can find an uncoloured vertex in $G_{1}$ and colours it. Thus $\Gamma_{\mathrm{g}}^{A}(2 G)>k-1$. If $G$ has an even number of vertices, the same argument as above shows that Bob has a winning strategy for the A-colouring game on $(2 G)^{+}$. This proves that $\Gamma_{\mathrm{g}}^{A}(\mathcal{H}) \geq k$.

$\mathrm{RR} \mathrm{n}^{\circ} 7646$ 
Assume $\Gamma_{\mathrm{g}}^{A}(\mathcal{H})=k$. Then there is a graph $G \in \mathcal{H}$ such that $\Gamma_{\mathrm{g}}^{A}(G)>k-1$. Then for the greedy colouirng game on $G^{+}$, Bob can simply colour the added isolated vertex in his first move, and then use his winning strategy for the Acolouring game afterward. This proves that $\Gamma_{\mathrm{g}}^{B}(\mathcal{H}) \geq k$, and hence completes the proof of Proposition 3

In this paper, we study the game Grundy number of $\mathcal{F}$ and $\mathcal{P} \mathcal{T}_{2}$ which satisfies the hypothesis of Proposition 3. Hence, we will consider Alice-first game Grundy number, so we abbreviate it in game Grundy number. We also write $\Gamma_{\mathrm{g}}$ instead of $\Gamma_{\mathrm{g}}^{A}$.

Clearly, $\Gamma_{\mathrm{g}} \leq \operatorname{col}_{\mathrm{g}}$. Hence, since $\operatorname{col}_{\mathrm{g}}(\mathcal{F})=4$ and $\operatorname{col}_{\mathrm{g}}\left(\mathcal{P} \mathcal{T}_{2}\right)=8$, we have $\Gamma_{\mathrm{g}}(\mathcal{F})=4$ and $\Gamma_{\mathrm{g}}\left(\mathcal{P} \mathcal{T}_{2}\right) \leq 8$. In this paper, we improve these two bounds. We shall prove that $\Gamma_{\mathrm{g}}(\mathcal{F})=3$ and $\Gamma_{\mathrm{g}}\left(\mathcal{P} \mathcal{T}_{2}\right) \leq 7$. We first prove in Theorem 6 that the game Grundy number of a forest is at most 3 and then show in Remark 7 trees whose game Grundy number is 3 . Finally we prove and the game Grundy number of a partial 2-tree is at most 7 . We do not know if there are partial 2-trees with game Grundy number 7.

By definition, $\chi(G) \leq \Gamma(G)$. But the similar inequality does not hold for game numbers, because $\chi_{\mathrm{g}}(G)$ may be bigger than $\Gamma_{\mathrm{g}}(G)$. Indeed $\chi_{\mathrm{g}}(\mathcal{F})=4$ and $\Gamma_{\mathrm{g}}(\mathcal{F})=3$. Hence a natural question is to ask if the game chromatic number can be very large compared to the game Grundy number. In Section 4, we answer by the affirmative by showing graphs $G_{k}, k \geq 2$, such that $\Gamma_{\mathrm{g}}\left(G_{k}\right)=k$ and $\chi_{\mathrm{g}}\left(G_{k}\right)=2^{k-1}+1$. However we do no not know whether the the game chromatic number can be bounded by a function of the game Grundy number.

Problem 4. Does there exists a function $f$ such that, $\chi_{\mathrm{g}}(G) \leq f\left(\Gamma_{\mathrm{g}}(G)\right)$ for all graph $G$ ?

On the opposite, we do not know if the Grundy game number can be bigger than the game chromatic number or not.

Problem 5. Is it true that $\Gamma_{\mathrm{g}}(G) \leq \chi_{\mathrm{g}}(G)$ for all graph $G$ ? And, if not, can $\Gamma_{\mathrm{g}}$ be arbitrarily large compared to $\chi_{\mathrm{g}}$ ?

\section{Game Grundy number of a forest}

Theorem 6. If $F$ is a forest, then $\Gamma_{\mathrm{g}}(F) \leq 3$.

Proof. Let us first start with few definitions. A vertex of a forest is called a leaf if it has degree at most 1 and a node if it has degree at least 3. A vertex which is not a leaf is an inner vertex.

Given a partial colouring $c$ of a forest $F$, the c-components, or simply components, are the inclusion-wise maximal subtrees such that no inner vertex is coloured, i.e. such that every coloured vertex is a leaf. A component is empty if it is an edge and its two vertices are coloured. If $C$ is a component, then its core is its minimum subtree containing all its coloured leaves.

A component $C$ is good if it is of one of the following types. 
Type I: Exactly two leaves are coloured in $\{2,3\}$, these leaves are at distance different from 2 and no other leaves are coloured.

Type II: Exactly one leaf is coloured in $\{2,3\}$ and the core of $C$ has at most one node. Moreover, if one leaf is coloured 3 and $C$ has a node, then the leaf of colour 3 is not adjacent to the node.

Type III: No leaves are coloured in $\{2,3\}$ and the core of $C$ has at most two nodes.

Type IV: No leaf is coloured 3, at least two leaves are coloured 2 and the core of $C$ has a unique node which is adjacent to all leaves coloured 2.

A component which is not good is said to be bad.

It is simple matter to see that if a player chooses a vertex in a good component then it is coloured in $\{1,2,3\}$. Indeed to be coloured at least 4 , it must be adjacent to three coloured vertices, two of which are coloured in $\{2,3\}$.

Let us show that Alice can play so that after each of her turns, all the components are good. Trivially at the beginning all the components are of Type III.

Suppose first that after Bob plays, all the components are good. We will show that Alice can play on a component so that no bad component is created.

- Suppose that there exists a nonempty component of Type I. Then the distance between the two coloured leaves is at least 3. Alice plays on the neighbour of one of these leaves. This vertex will be coloured 1 , because its only coloured neighbour is coloured in $\{2,3\}$. Hence all the new components are good.

- Suppose now that there exists a nonempty component $C$ of Type II. Let $v$ be the leaf coloured in $\{2,3\}$. Alice colours the neigbour $w$ of $v$ in the core (or any neighbour of $v$ if the core is restricted to $v$ ). If vertex $w$ gets colour 1, all the nontrivial new components are of Type III. If not, then $w$ is coloured in $\{2,3\}$ and must be adjacent to a leaf of $C$ coloured 1 . In particular, if the core of $C$ has a node, it is $w$. Hence, all the nonempty new components are of Type II.

- Suppose that there exists a nonempty component $C$ of Type III. Alice plays on a node of a core if one exists or on any neighbour of a leaf if there is no. The chosen vertex $w$ will be coloured either 1 or 2 and the cores of the new components will have at most one node each. Hence all the new components are of type II or III.

- Suppose finally that there exists a component $C$ of Type IV. Alice plays on the node of its core. This vertex is then coloured 1 or 3 and all the nonempty new components are of type II or III.

$\mathrm{RR} \mathrm{n}^{\circ} 7646$ 
Suppose now that after Bob's play, a bad component was created. We distinguish four cases according to the type of the component onto which Bob has played.

1) Assume that Bob played on a component $C$ of Type I. Let $u$ and $v$ be the two coloured leaves of $C$. The core of $C$ is the path $P$ from $u$ to $v$. Observe that Bob has not played on a vertex of $P$, otherwise all the created components would be good. Hence, Bob played on a vertex $w$ outside $P$. Then $w$ is coloured 1 . All the created components not containing $u$ and $v$ are trivially of Type III, as their unique coloured leaf is $w$. Let us now consider the component $C^{\prime}$ containing $u, v$ and $w$. Since $P$ has length at least 3 , then one vertex of $\{u, v\}$, say $u$, is not adjacent to the node of the core of $C^{\prime}$. Alice colours its neighbour $z$. It will be coloured 1, and thus $C^{\prime}$ is cut into an empty component, a component of Type II, (the one containing $z, v$ and $w$ ) and all the others are of Type III, since their unique coloured leaf is $z$.

2) Assume that Bob played on a component $C$ of Type II.

Let $u$ be the vertex of $C$ coloured in $\{2,3\}$.

- Assume Bob played on a vertex $w$ outside the core of $C$. Then $w$ is coloured 1. Moreover, $C$ is cut into components of type III and one component $C^{\prime}$ containing $w$ and all the coloured leaves of $C$. Let $t$ be the neighbour of $u$ in the core of $C^{\prime}$. If $t$ has no neighbour coloured 1 , then Alice plays on $t$. Then $t$ is coloured 1 , so all the new nonempty components are of type III. Assume now that $t$ has a neighbour coloured 1, then $t$ is one of the two nodes of the core of $C^{\prime}$. If $u$ is coloured 3 (resp. $u$ is coloured 2 and $t$ is not adjacent to the second node of the core of $C^{\prime}$ ), then Alice plays on $t$. Then $t$ is coloured 2 (resp. 3), so all the new nonempty components are of type II. Finally if $u$ is coloured 2 and $t$ is adjacent to the second node $s$ of the core of $C^{\prime}$, then Alice plays on $s$. It will be coloured either 1 or 2 since it can only be adjacent to vertices of colour 1 . Hence all the new components are of type IV, III or II.

- Assume Bob played on a vertex $w$ in the core of $C$.

Then $w$ cannot be coloured 1 , because a bad component must be created. It cannot be coloured 3 neither. Indeed it were, it would be adjacent to $u$ and another leaf coloured 1 of $C$. So $w$ would be node of the core of $C$, and all the nonempty new components would be of type II.

Hence $w$ is coloured 2. Then it must on the path between the node $t$ of the core of $C$ and a leaf distinct from $u$. In this case, the unique bad component $C^{\prime}$ is the one that contains $u$ and $w$. If one of $u$ and $w$ has a neighbour $z$ which is not the node of $C^{\prime}$ then Alice colours $z$. The vertex $z$ is coloured 1 , so all the new components are of Type II or I. If not, then $u$ and $w$ are both adjacent to $t$. In particular, 
$u$ is coloured 2, by definition of Type II component. Alice plays on $t$, which must be coloured 1 or 3 , because it is adjacent to no vertex coloured 3. Hence, all the nonempty new components are of type II or III.

3) Assume that Bob played on a component $C$ of Type III.

- Assume Bob played on a vertex $w$ outside the core of $C$. Then $w$ is coloured 1. The created bad component $C^{\prime}$ must have three nodes in its core. Alice plays on the node $t$ which is in the middle, i.e. on the path joining the two others. The vertex $t$ can only be adjacent to vertices coloured 1 , so it is coloured in $\{1,2\}$, and all the new components have at most one node. So they are of type II or III.

- Assume Bob played on a vertex $w$ in the core of $C$. Then $w$ cannot be coloured 1 otherwise all the components are of type III. So $w$ is coloured 2, because it is adjacent to no vertex coloured 2. Thus, the bad component $C^{\prime}$ obtained after Bob's play must contain $w$ and have two nodes $t_{1}$ and $t_{2}$ in its core.

If $w$ is adjacent to none of these nodes, then Alice plays in the neighbour $z$ of $w$ in the core of $C^{\prime}$. Then $w$ must be coloured 1 and all the new components are of type III.

Assume now that $w$ is adjacent to one of the nodes say $t_{1}$. If $t_{1}$ is not adjacent to $t_{2}$, then Alice plays on $t_{1}$. The vertex $t_{1}$ is coloured 3 or 1 and all the new components are of type II or III. If $t_{1}$ and $t_{2}$ are adjacent, then Alice plays on $t_{2}$. The vertex $t_{2}$ is then coloured 1 or 2 , because it is adjacent to no vertex coloured 2. Hence all the new components are of type II, III and possibly one is of type IV.

4) Assume that Bob played on a component $C$ of Type IV.

- Assume Bob played on a vertex $w$ outside the core of $C$. Then $w$ is coloured 1. If one bad component $C^{\prime}$ has been created, then it must have two nodes in its core. One of them $t_{1}$ is the one of the core of $C$ and thus is adjacent to all the leaves coloured 2. Let $t_{2}$ be the second node of the core of $C^{\prime}$. If $t_{1}$ is not adjacent to $t_{2}$, then Alice plays on it. So $t_{1}$ gets either colour 1 or 3 , because it is adjacent to no vertex coloured 3 and to vertices coloured 2.. Hence all the nonempty new components are of type III or II. If $t_{1}$ is adjacent to $t_{2}$, then Alice plays on $t_{2}$. This vertex gets colour 1 or 2 , because it is adjacent to no vertex coloured 3 . Hence the new component containing $t_{1}$ is still of type IV, and all the other new ones are of type II or III.

- Assume Bob played on a vertex $w$ in the core of $C$. Then $w$ is not coloured 1 because a bad component must be created. It cannot be coloured 3 neither, for otherwise it must be the node of the core of $C$, and so all the nonempty created components are of type II.

$\mathrm{RR} \mathrm{n}^{\circ} 7646$ 
Hence $w$ is coloured 2. Thus it is not adjacent to the node $t$ of the core of $C$, because a bad component must be created. Alice plays on $t$, which gets coloured 1 or 3 . Then all the nonempty new components are of type II or III.

Remark 7. The bound 3 in Theorem 6 is best possible, because there are trees $T$ for which $\Gamma_{\mathrm{g}}^{A}(T)=3$ and $\Gamma_{\mathrm{g}}^{B}(T)=3$. Consider for example the path on seven vertices $P_{7}=\left(v_{1}, \ldots, v_{7}\right)$.

Suppose that Alice plays first. Without loss of generality she played on some $v_{i}$ with $i \leq 4$. Then Bob plays on $v_{i+3}$. In the remainder of the game, the first chosen vertex among $\left\{v_{i+1}, v_{i+2}\right\}$ will be coloured 2 and the second one will be coloured 3. Hence $\Gamma_{\mathrm{g}}^{A}\left(P_{7}\right)=3$.

Suppose now that Bob plays first. Then he plays on $v_{4}$. Without loss of generality, we may assume that Alice played on $v_{i}$ for $1 \leq i \leq 3$. Bob plays on $v_{7}$. Similarly, the two vertices $\left\{v_{5}, v_{6}\right\}$ will be coloured 2 and 3 .

\section{Game Grundy number of a partial 2-tree}

A $k$-tree is defined inductively as follows: $K_{k+1}$ is a $k$-tree and if $G$ is a $k$-tree then every graph obtained from $G$ by adding a vertex and linking it to the $k$ vertices of a clique of $G$ is a $k$-tree. Obviously, the class of 1 -trees is the one of trees. To every $k$-tree, one can associate the ordering of the vertices $\left(v_{1}, \ldots, v_{n}\right)$ corresponding to the order in which the vertices are added in the inductive construction. Hence $\left\{v_{1}, \ldots, v_{k+1}\right\}$ is the original clique and for every $i \geq k+1$, vertex $v_{i}$ has exactly $k$ left neighbours which form a clique (we think of the vertices as line up from left to right).

A partial $k$-tree is a subgraph of a $k$-tree.

Theorem 8. If $G$ is a partial 2-tree, then $\Gamma_{\mathrm{g}}(G) \leq 7$.

Proof. Let $G$ be a partial 2-tree and let $H$ be a 2-tree containing $G$ and $\left(v_{1}, \ldots, v_{n}\right)$ be the ordering associated to $H$. We consider an ordering as a placement on a line from left to right. Hence a vertex is left (resp. right) to another it is has smaller (resp. larger) index.

The strategy used by Alice is the one described in $\left[18\right.$ to prove that $\operatorname{col}_{\mathrm{g}}(G) \leq$ 8. Alice will record a set of active vertices. A coloured vertex is necessarily activated, but some activated vertices are not coloured. An uncoloured vertex will become active only if it is activated by Alice as described below. In particular, no vertex is active at the beginning. Suppose that Bob plays on a vertex $v$. Then Alice plays according to the following algorithm. All the considered adjacencies are in $H$.

0. If all its left neighbours are coloured, then Alice plays on the leftmost vertex with smallest index, otherwise let $u:=v$. 
1. Let $w$ be the uncoloured left neighbour of $u$ with smallest index.

2. If $w$ is activated or its two left neighbours are already coloured, then Alice colours $u$. Otherwise, activate $u$ and let $u:=w$ and Go to Step 1 .

When we do the operation $u:=w$ in Step 2, we say that Alice jumps from $u$ to $w$. Hence the above algorithm may be seen as follows: Alice jumps from a vertex to its leftmost uncoloured left neighbour until she cannot jump anymore. She activates all the vertices onto which she jumps, and colour the one on which she stops.

When Alice jumps from vertex $u$ to vertex $w$, we orient the edge $u w$ from $u$ to $w$. At the end of each move of Alice, a set $F$ of edges of $H$ is oriented (oriented edges remain oriented afterwards). Set $D=(V(G), F)$.

Observe that Alice jumps at most once from a vertex and at most twice onto a vertex and that she colours the vertex the second time she jumps onto it. In particular, every vertex of $D$ has outdegree at most 1 and indegree at most 2 . In addition, if a vertex has indegree 2, then its outdegree is 1 .

For every vertex $x$, let $P(x)$ be the set of neighbours of $x$ that are coloured previously to $x$, and let $R(x)$ be the set of vertices in $P(x)$ right to $x$.

Claim 1. Let $x$ be a vertex with left neighbours $x_{1}$ and $x_{2}$. Then $R(x)=R^{\prime}(x) \cup$ $R^{\prime \prime}(x)$ with $R^{\prime}(x)=R(x) \cap N^{-}(x)$ and $R^{\prime \prime}(x)=R(x) \cap\left(N^{-}\left(x_{1}\right) \cup N^{-}\left(x_{2}\right)\right)$.

Proof. Let $z$ be a vertex in $R(x)$. If Alice coloured $z$, then she did it when jumping a second time on it, because one of its left neighbour, namely $x$, is uncoloured. Then the first time Alice jumped onto $z$, she has jumped again from $z$ onto another vertex. If Bob coloured $z$, then Alice has jumped to one of its left neighbour. So, in both cases, Alice has jumped from $z$. If Alice did not jump from $z$ to $x$, then she jumped to a vertex left to $x$. That vertex must be adjacent to $x$ since the left neighbourhood of every vertex is a clique in $H$. Hence Alice jumped from $z$ to either $x_{1}$ or $x_{2}$.

Claim 2. For every vertex $x$, we have $\left|R^{\prime}(x)\right| \leq 2, \mid R^{\prime \prime}(x) \leq 4$ and $|R(x)| \leq 5$.

Proof. Alice jumps at most twice on each vertex, so $\left|R^{\prime}(x)\right| \leq 2$ and $\left|R^{\prime \prime}(x)\right| \leq 4$. Furthermore, if $\left|R^{\prime}(x)\right|=2$, then $\left|R^{\prime \prime}(x)\right| \leq 3$, because the first time Alice jumped onto $x$, she did not colour it. So she must have jumped onto one of its left neighbours. Hence at most three of the vertices from which Alice jumped onto $\left\{x_{1}, x_{2}\right\}$ are distinct from $x$. It follows that $|R(x)| \leq 5$.

Suppose by way of contradiction that there exists a vertex $v$ coloured 8 . Let $v_{1}$ and $v_{2}$ be its two left neighbours with $v_{2}$ left to $v_{1}$. Necessarily, $|P(v)| \geq 7$. Hence, since $R(v)=P(v) \backslash\left\{v_{1}, v_{2}\right\}$, Claim 2 yields $|R(v)|=5$ and $\left\{v_{1}, v_{2}\right\} \subset$ $P(v)$.

Let $q$ be the vertex of $R^{\prime}(v)$ which is coloured last and let $p$ be the other vertex of $R^{\prime}(v)$ if it exists. For convenience and with a slight abuse of notation, $S \backslash\{p\}$ will denote the set $S$ if $p$ does not exists.

$\mathrm{RR} \mathrm{n}^{\circ} 7646$ 
Claim 3. Bob coloured $q$ right before Alice coloured $v$ and $R^{\prime}(q)=\emptyset$.

Proof. We claim that Alice coloured $v$ right after jumping from $q$. Indeed if $\left|R^{\prime}(v)\right|=2$, then Alice have jumped twice on $v$ and thus Alice has coloured $v$ jumping from $q$. If $\left|R^{\prime}(v)\right|=1$, then, as $|R(v)|=5$, Claim 2 yields $\left|R^{\prime \prime}(v)\right|=4$. Thus Alice has not jumped from $v$ to $x_{1}$ or $x_{2}$, and so she coloured $v$ the first time she jumped onto it. But to $v$, she only jumped from $q$.

Now observe that Alice never jumps from a coloured vertex unless Bob just coloured it. So Bob coloured $q$ and Alice did not. Since Alice jump at most once from a vertex, she has not jumped from $q$ before colouring it. Therefore she has not jumped onto $q$ before colouring it, otherwise she would have jumped, because $v$ was still available. Hence $R^{\prime}(q)=\emptyset$.

Claim 4. If $w \in R^{\prime \prime}(v)$, then $|R(w) \backslash\{p\}| \leq 2$.

Proof. Let $w \in R^{\prime \prime}(v)$. Then its left neighbours are $v$ and the outneighbour $v^{\prime} \in\left\{v_{1}, v_{2}\right\}$ of $w$. Let $z$ be in $R^{\prime \prime}(w)$. Alice must have jumped from $z$ to a left neighbour of $z$ left from $w$. Since the left neighbourhoods are cliques, it must be onto $v$ or $v^{\prime}$. Let $x_{1}, x_{2}$ be the two inneighbours of $v^{\prime}$. Since $|R(v)|=5$, we conclude that $v$ and $v^{\prime}$ are the two left neighbours of $x_{1}, x_{2}$. Hence $w$ is not a left neighbour of $x_{1}$ or $x_{2}$. Moreover $q$ was not coloured when $w$ is coloured by Claim 3. Hence $R^{\prime \prime}(w) \subset\{p\}$. By Claim 2, we get $|R(w) \backslash\{p\}| \leq 2$.

Claim 5. $\left|R^{\prime \prime}(v)\right|=3$.

Proof. Suppose for a contradiction that $\left|R^{\prime \prime}(v)\right|=4$. Then by Claim $4,|R(w)| \leq$ 2 for all $w \in R^{\prime \prime}(v)$. The left neighbourhood of every vertex $w$ of $R^{\prime \prime}(v)$ is $\left\{v, v_{w}^{\prime}\right\}$ with $v_{w}^{\prime} \in\left\{v_{1}, v_{2}\right\}$ and so $P(w) \subset R(w) \cup\left\{v_{w}^{\prime}\right\}$.

Assume that no vertex of $\left\{v_{1}, v_{2}\right\}$ is coloured in $\{1,2,3\}$. For each $w \in R^{\prime \prime}(v)$, a colour of $\{1,2,3\}$ is not assigned to any of its coloured neighbour. Hence $w$ is coloured at most 3 . Therefore, when Alice coloured $v$, this vertex had at most three neighbours $\left(v_{1}, v_{2}\right.$ and $\left.q\right)$ coloured in $\{4,5,6,7\}$. So she coloured it with a colour smaller than 8 , a contradiction.

Assume now that at least one vertex $v^{\prime}$ of $\left\{v_{1}, v_{2}\right\}$ is coloured in $\{1,2,3\}$. Every vertex $w$ of $R^{\prime \prime}(v)$ is coloured at most 4 , since $|P(w)| \leq 3$. Therefore, when Alice coloured $v$, this vertex had at most two neighbours ( $q$ and the one of $\left.\left\{v_{1}, v_{2}\right\} \backslash\left\{v^{\prime}\right\}\right)$ coloured in $\{5,6,7\}$. So she coloured it with a colour smaller than 8 , a contradiction.

Claim 6. $|P(q) \backslash\{p\}| \leq 3$.

Proof. Let $r$ be the left neighbour of $q$ distinct from $v$. By Claim $3, R^{\prime}(q)=\emptyset$. Hence a vertex in $P(q) \backslash\{p\}$ is either $r$ or one of the at most two inneighbours of $r$.

Claim 7. $|P(p)| \leq 5$.

Proof. Let $r$ be left neighbour of $p$ distinct from $v$. The vertices $q$ and $v$ are coloured after $p$, thus $P(p) \subset\{r\} \cup N^{-}(r) \cup N^{-}(q)$. So $|P(p)| \leq 5 \mid$. 
Let $v_{3}$ be the left neighbour of $v_{1}$ distinct from $v_{2}$.

Claim 8. $\left|P\left(v_{1}\right) \backslash\left\{v_{2}\right\}\right| \leq 4$. Moreover if $\left|P\left(v_{1}\right) \backslash\left\{v_{2}\right\}\right|=4$, then $v_{2}$ is an outneighbour of $v$ and the inneighbour $t_{3}$ of $v_{3}$ distinct from $v_{1}$ is in $R\left(v_{1}\right)$.

Proof. By Claim $5 .\left|R^{\prime}(v)\right|=2$, so $N^{-}(v)=2$ and thus $N^{+}(v)=1$. So Alice has jumped from $v$ to one of its left neighbours. Hence $\left|N^{-}\left(v_{1}\right) \cup N^{-}\left(v_{2}\right)\right|=\mid R^{\prime \prime}(v) \cup$ $\{v\} \mid=4$ and so $\left|N^{-}\left(v_{1}\right)\right|=\left|N^{-}\left(v_{2}\right)\right|=2$. Therefore $\left|N^{+}\left(v_{1}\right)\right|=\left|N^{+}\left(v_{2}\right)\right|=1$. The outneighbour of $v_{1}$ is necessarily $v_{3}$ because the inneighbours of $v_{2}$ are in $R^{\prime \prime}(v) \cup\{v\}$.

Now $P\left(v_{1}\right) \subset\left\{v_{2}, v_{3}\right\} \cup N^{-}\left(v_{1}\right) \cup N^{-}\left(v_{2}\right) \cup N^{-}\left(v_{3}\right)$. But every vertex in $N^{-}\left(v_{2}\right)$ is either $v$ or has $v$ and $v_{2}$ as left neighbours. In both cases it is not a right neighbour of $v_{1}$. Therefore $P\left(v_{1}\right) \subset\left\{v_{2}, v_{3}\right\} \cup N^{-}\left(v_{1}\right) \cup N^{-}\left(v_{3}\right)$ and so $P\left(v_{1}\right) \subset\left\{v_{2}, v_{3}, t_{3}\right\} \cup N^{-}\left(v_{1}\right)$ because $N^{-}\left(v_{3}\right)=\left\{v_{1}, t_{3}\right\}$. Hence, $\left|P\left(v_{1}\right) \backslash\left\{v_{2}\right\}\right| \leq$ 4 .

Moreover, if there is equality, then $t_{3}$ and the two vertices of $N^{-}\left(v_{1}\right)$ are in $P\left(v_{1}\right)$. Thus $v$ is not in $N^{-}\left(v_{1}\right)$, because it is not in $P\left(v_{1}\right)$ for it is coloured after $v_{1}$. As $v$ is in $N^{-}\left(v_{1}\right) \cup N^{-}\left(v_{2}\right)$, it is an inneighbour of $v_{2}$.

Claim 9. $v_{2}$ is coloured 7, $p$ is coloured 6, $v_{1}$ is coloured 5, $q$ is coloured 4 and the vertices of $R^{\prime \prime}(v)$ are coloured in $\{1,2,3\}$.

Proof. By Claims 4, 6, 7 and 8, every vertex $x$ of $P(v) \backslash\left\{v_{2}\right\}$ satisfies $|P(x)| \leq 5$ and thus is coloured at most 6 . Hence $v_{2}$ is coloured 7 . Now every vertex $x$ of $P(v) \backslash\left\{p, v_{2}\right\}$ satisfies $\left|P(x) \backslash\left\{v_{2}\right\}\right| \leq 4$ and thus is coloured at most 5 . Hence $p$ is coloured 6. Furthermore, every vertex $x$ of $P(v) \backslash\left\{p, v_{1}, v_{2}\right\}$ satisfies $\left|P(x) \backslash\left\{p, v_{2}\right\}\right| \leq 3$ and thus is coloured at most 4. Hence $v_{1}$ is coloured 5 . Finally, every vertex $w$ of $R^{\prime \prime}(v)$ satisfies $\left|P(w) \backslash\left\{p, v_{1}, v_{2}\right\}\right| \leq 2$ and thus is coloured at most 3. Hence $q$ is coloured 4 .

Let $y_{1}$ and $y_{2}$ be the two left neighbours of $v_{2}$. Since $N^{-}\left(v_{2}\right)=2$, one of these vertices, say $y_{1}$ is an outneighbour of $v_{2} . P\left(v_{2}\right) \subset\left\{y_{1}, y_{2}\right\} \cup N^{-}\left(y_{1}\right) \cup$ $N^{-}\left(y_{2}\right) \cup N^{-}\left(v_{2}\right)$. By Claim 9, $v_{1}$ is coloured 5, so $\left|P\left(v_{1}\right)\right| \geq 4$. Thus, by Claim 8, $v$ is an inneighbour of $v_{2}$. Let $w$ be the inneighbour of $v_{2}$ distinct from $v$. Then $w \in R^{\prime \prime}(v)$ and thus $w$ is coloured at most 3 .

Now $P\left(v_{2}\right) \subset\left\{y_{1}, y_{2}, w\right\} \cup N^{-}\left(y_{1}\right) \cup N^{-}\left(y_{2}\right)$, because $v$ is coloured after $v_{2}$. Moreover since $v_{2} \in N^{-}\left(y_{1}\right) \cup N^{-}\left(y_{2}\right)$, this yields $\left|P\left(v_{2}\right)\right| \leq 6$. But $v_{2}$ is coloured 7 by Claim 9, so $\left|P\left(v_{2}\right)\right|=6$. In addition, the three inneighbours of $y_{1}$ and $y_{2}$ different from $v_{2}$ are in $R^{\prime \prime}\left(v_{2}\right)$. This implies that $v_{3} \notin\left\{y_{1}, y_{2}\right\}$ because by Claim $8, t_{3}$ is not in $R^{\prime \prime}\left(v_{2}\right)$ since its two left neighbours are $v_{1}$ and $v_{3}$.

Set $Z=\left(N^{-}\left(y_{1}\right) \cup N^{-}\left(y_{2}\right)\right) \backslash\left\{v_{2}\right\}$.

Claim 10. If $z$ is a vertex of $Z$, then $R^{\prime \prime}(z)=\emptyset$.

Proof. Assume for a contradiction that there exists a vertex $t \in R^{\prime \prime}(z)$. Then it must be an inneighbour of $v_{2}$ or $y_{i}$ for some $i \in\{1,2\}$. But an inneighbour of $y_{i}$ (distinct from $v_{2}$ ) has $v_{2}$ and $y_{i}$ as left neighbours and thus is not a right neighbour of $z$. And an inneighbour of $v_{2}$ is either $v$ which is coloured after $z$, 
or a vertex of $R^{\prime \prime}(v)$ which is not a right neighbour of $z$ since its left neighbours are in $\left\{v_{1}, v_{2}, v\right\}$.

We now get a contradiction in a similar way as the proof of Claim 5 .

Assume first that no vertex of $\left\{y_{1}, y_{2}\right\}$ is coloured in $\{1,2,3\}$. For each $z \in Z$, a colour of $\{1,2,3\}$ is not assigned to any of its coloured neighbour. Hence $z$ is coloured at most 3. Therefore, when Alice coloured $v_{2}$, this vertex had at most two neighbours coloured in $\{4,5,6\}$. So she coloured it with a coloured smaller than 7 , a contradiction.

Assume finally that at least one vertex $y^{\prime}$ of $\left\{y_{1}, y_{2}\right\}$ is coloured in $\{1,2,3\}$. Every vertex $z$ of $Z$ is coloured at most 4 , since $|P(z)| \leq 3$. Therefore, when Alice coloured $v_{2}$, this vertex had at most one neighbour (the one of $\left\{y_{1}, y_{2}\right\} \backslash$ $\left\{y^{\prime}\right\}$ ) coloured in $\{5,6\}$, namely $y_{1}$ and $y_{2}$. So she coloured $v_{2}$ with a colour smaller than 7 , a contradiction.

\section{Graphs with game chromatic number larger than game Grundy number}

For two graphs the disjoint union of two copies of $G_{1}$ and $G_{2}$ is denoted $G_{1}+G_{2}$. The join of $G_{1}$ and $G_{2}$ is the graph $G_{1} \oplus G_{2}$ obtained from $G_{1}+G_{2}$ by joining all the vertices of the copy of $G_{1}$ to all the vertices of the copy of $G_{2}$. For a positive integer $p$, we denote by $p G$ the disjoint union of $p$ copies of $G$.

A cograph is a graph without induced subgraph isomorphic to $P_{4}$, the path on four vertices. The family of cographs may also be defined inductively as follows:

- $K_{1}$ is a cograph;

- if $G_{1}$ and $G_{2}$ are cographs, then $G_{1}+G_{2}$ and $G_{1} \oplus G_{2}$ are cographs.

It is well known that if $G$ is a cograph, then $\chi(G)=\Gamma(G)$. Since $\chi(G) \leq$ $\Gamma_{\mathrm{g}}(G) \leq \Gamma(G)$, we have the following.

Proposition 9. If $G$ is a cograph, then $\chi(G)=\Gamma_{\mathrm{g}}(G)=\Gamma(G)$.

Theorem 10. For integer $k \geq 2$, there exists a graph $G_{k}$ such that $\Gamma_{\mathrm{g}}\left(G_{k}\right)=k$ and $\chi_{\mathrm{g}}\left(G_{k}\right) \geq 2^{k-1}+1$.

Proof. We will construct by induction a sequence $\left(G_{k}\right)$ of cographs satisfying $\Gamma_{\mathrm{g}}\left(G_{k}\right)=k$ and $\chi_{\mathrm{g}}\left(G_{k}\right) \geq 2^{k}-1$.

Let $p_{k}=2^{k-1}+1$. Observe that $p_{2}=3$ and $p_{k}=2 p_{k-1}-1$. For any integer $q$, we denote by $S_{q}$ the graph with $q$ vertices and no edges.

$G_{2}$ is the cycle of length 4 . It is a cograph and $\Gamma_{\mathrm{g}}\left(G_{2}\right)=\chi\left(G_{2}\right)=2$ and $\chi_{\mathrm{g}}\left(G_{2}\right)=3$.

For every $k \geq 2$, let $G_{k+1}=p_{k} G_{k} \oplus S_{2 p_{k}-2}$. A simple induction implies that every $G_{k}$ has an even number of vertices because $G_{2}$ does.

Moreover every $G_{k}$ is a cograph since it obtained from cographs by a series of disjoint union and joins. Hence $\Gamma_{\mathrm{g}}\left(G_{k}\right)=\chi\left(G_{k}\right)$ by Proposition 9 . In 
addition, since for any graphs $G$ and $H$ and integer $p, \chi(p G)=\chi(G)$ and $\chi(G \oplus H)=\chi(G)+\chi(H)$, it follows that $\chi\left(G_{k+1}\right)=\chi\left(G_{k}\right)+1$. Hence by induction, $\Gamma_{\mathrm{g}}\left(G_{k}\right)=\chi\left(G_{k}\right)=k$ for all $k \geq 2$.

Let us now prove by induction that $\chi_{\mathrm{g}}\left(G_{k}\right) \geq p_{k}$ for all $k \geq 2$, the result holding for $k=2$. Let $k \geq 2$. Since $\chi_{\mathrm{g}}\left(G_{k}\right)>p_{k}-1$, Bob has a strategy, called BWIN, to win the colouring game on $G_{k}$ with colour set $\left\{1, \ldots, p_{k}-1\right\}$. Let us describe a strategy so that Bob wins on $G_{k+1}$ with $\left\{1, \ldots, p_{k+1}-1\right\}$. In the first $p_{k}-1$ of its moves, Bob assign $p_{k}-1$ different colours, say those of $\left\{p_{k}, \ldots, p_{k+1}-1\right\}$, to different vertices of the copy of $S_{2 p_{k}-2}$. This is always possible because at most $2 p_{k}-3$ vertices are played on before Bob plays its $\left(p_{k}-1\right)^{\text {th }}$ move. After these first moves all the colours in $\left\{p_{k}, \ldots, p_{k+1}-1\right\}$ are used on the copy $S_{p_{k}-2}$, so only colours in $\left\{1, \ldots, p_{k}-1\right\}$ may be used when playing on vertices of $p_{k} G$. Note moreover that before Bob's $\left(p_{k}-1\right)^{\text {th }}$ move, Alice has played at most $p_{k}-1$ times, so one of the copies of $G_{k}$ in $p_{k} G$, say $G^{*}$, as not been played on. The strategy of Bob from its $p^{\text {th }}$ move on will then be the following: if Alice does not play on $G^{*}$, then Bob plays also on a vertex not in $G^{*}$ (This is possible because $G_{k+1}-G^{*}$ has an even number of vertices.); if Alice plays on $G^{*}$, then Bob plays also on $G^{*}$ according to the strategy BWIN. Thus all the vertices of $G^{*}$ will not be coloured and Bob wins. Hence $\chi_{\mathrm{g}}\left(G_{k}\right)>p_{k+1}-1$.

\section{References}

[1] T. Bartnicki, J. Grytczuk, H. A. Kierstead, and X. Zhu. The map colouring game. American Mathematics Monthly, to appear.

[2] H. L. Bodlaender. On the complexity of some coloring games. Internat. J. Found. Comput. Sci., 2(2):133-147, 1991.

[3] C. Dunn and H. A. Kierstead. A simple competitive graph coloring algorithm. II. J. Combin. Theory Ser. B, 90(1):93-106, 2004. Dedicated to Adrian Bondy and U. S. R. Murty.

[4] C. Dunn and H. A. Kierstead. A simple competitive graph coloring algorithm. III. J. Combin. Theory Ser. B, 92(1):137-150, 2004.

[5] U. Faigle, U. Kern, H. A. Kierstead, and W. T. Trotter. On the game chromatic number of some classes of graphs. Ars Combin., 35:143-150, 1993.

[6] M. Gardner. Mathematical games. Scientific American, April, 1981.

[7] D. J. Guan and X. Zhu. Game chromatic number of outerplanar graphs. J. Graph Theory, 30(1):67-70, 1999.

$\mathrm{RR} \mathrm{n}^{\circ} 7646$ 
[8] W. He, X. Hou, K.-W. Lih, J. Shao, W. Wang, and X. Zhu. Edge-partitions of planar graphs and their game coloring numbers. J. Graph Theory, 41(4):307-317, 2002.

[9] H. A. Kierstead. A simple competitive graph coloring algorithm. J. Combin. Theory Ser. B, 78(1):57-68, 2000.

[10] H. A. Kierstead and W. T. Trotter. Planar graph coloring with an uncooperative partner. J. Graph Theory, 18(6):569-584, 1994.

[11] H. A. Kierstead and W. T. Trotter. Competitive colorings of oriented graphs. Electron. J. Combin., 8(2):Research Paper 12, 15 pp. (electronic), 2001. In honor of Aviezri Fraenkel on the occasion of his 70th birthday.

[12] H. A. Kierstead and Zs. Tuza. Marking games and the oriented game chromatic number of partial $k$-trees. Graphs Combin., 19(1):121-129, 2003.

[13] H. A. Kierstead and D. Yang. Orderings on graphs and game coloring number. Order, 20(3):255-264 (2004), 2003.

[14] H. A. Kierstead and D.Yang. Very asymmetric marking games. Order, 22(2):93-107, 2005.

[15] J. Nešetřil and E. Sopena. On the oriented game chromatic number. Electron. J. Combin., 8(2):Research Paper 14, 13 pp. (electronic), 2001. In honor of Aviezri Fraenkel on the occasion of his 70th birthday.

[16] J. Wu and X. Zhu. Lower bounds for the game colouring number of partial $k$-trees and planar graphs. Dsicrete Mathematics 308(12):2637-2642, 2008.

[17] X. Zhu. The game coloring number of planar graphs. J. Graph Theory, 59:261-278, 2008.

[18] X. Zhu. The game coloring number of pseudo partial $k$-trees. Discrete Math., 215(1-3):245-262, 2000.

[19] X. Zhu. Refined activation strategy for the marking game. J. Combin. Theory Ser. B 98(1):1-18, 2008.

[20] X. Zhu. Game colouring Cartesian product of graphs. J. Combin. Theory Ser. B 98(1):1-18, 2008. 


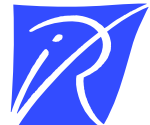

Centre de recherche INRIA Sophia Antipolis - Méditerranée 2004, route des Lucioles - BP 93 - 06902 Sophia Antipolis Cedex (France)

Centre de recherche INRIA Bordeaux - Sud Ouest : Domaine Universitaire - 351, cours de la Libération - 33405 Talence Cedex Centre de recherche INRIA Grenoble - Rhône-Alpes : 655, avenue de l'Europe - 38334 Montbonnot Saint-Ismier

Centre de recherche INRIA Lille - Nord Europe : Parc Scientifique de la Haute Borne - 40, avenue Halley - 59650 Villeneuve d'Ascq

Centre de recherche INRIA Nancy - Grand Est : LORIA, Technopôle de Nancy-Brabois - Campus scientifique 615, rue du Jardin Botanique - BP 101 - 54602 Villers-lès-Nancy Cedex

Centre de recherche INRIA Paris - Rocquencourt : Domaine de Voluceau - Rocquencourt - BP 105 - 78153 Le Chesnay Cedex

Centre de recherche INRIA Rennes - Bretagne Atlantique : IRISA, Campus universitaire de Beaulieu - 35042 Rennes Cedex

Centre de recherche INRIA Saclay - Île-de-France : Parc Orsay Université - ZAC des Vignes : 4, rue Jacques Monod - 91893 Orsay Cedex 